\title{
EDITORIAL AND COMMENT Eliciting Personal Values of Patients with Multiple Chronic Conditions: Why and How
}

\author{
Michele Heisler, MD, MPA ${ }^{1,2,3}$ \\ ${ }^{1}$ Center for Clinical Management Research, Ann Arbor Veterans Affairs (VA) Healthcare System, Ann Arbor, MI, USA; ${ }^{2}$ Department of Internal \\ Medicine, University of Michigan Medical School, Ann Arbor, MI, USA; ${ }^{3}$ Department of Health Behavior and Health Education, School of Public \\ Health, University of Michigan, Ann Arbor, MI, USA.
}

J Gen Intern Med 32(12): 1273-4

DOI: $10.1007 /$ s11606-017-4183-y

(c) Society of General Internal Medicine 2017

$\mathrm{T}$ hink about your own clinical practice. How often do you try to engage your patients in a discussion about what is most important to them for their well-being and health, beyond a narrow discussion of their preferences for specific diagnostic and treatment options? If you have broached the topic of core values, how often has the patient looked somewhat surprised and responded with a brief, medically focused answer (e.g., "I hate taking medications")? I suspect that, like me, many other clinicians rarely broach questions of personal values - defined as what a person considers important in life - and when we do, our patients understand us to mean medically related values and respond accordingly.

With the welter of demands on busy clinicians, why might we consider working to effectively initiate and engage in such discussions? One reason is our commitment to providing "patient-centered" care. Eliciting and incorporating patient values in the care we provide is a foundation of "patient centeredness." The Institute of Medicine defines patient centeredness as "care that is respectful of and responsive to individual patient preferences, needs, and values, and ensuring that patient values guide all clinical decisions." ${ }^{1}$ Central to patient-centered care is defining a "good" outcome in terms of what is meaningful and valuable to the individual patient. A careful exploration of what is most important to each of our patients is thus necessary to help work with them to achieve such outcomes. There is a growing body of evidence that better eliciting individuals' values during clinical encounters improves their experiences with health care and more effectively aligns treatments with goals of care; in addition, patients who rate their providers highly on patient centeredness are more likely to adhere to treatment recommendations. ${ }^{2}$ Also, eliciting patients' life goals and values is an essential part of efforts to help motivate positive health behavior changes. A key strategy in effective behavioral counseling approaches such as motivational interviewing is to encourage patients to

Published online September 18, 2017 reflect on how their current health behaviors contribute to or detract from what is most important to them in their lives (e.g., being a good parent). ${ }^{3}$ Linking healthy behaviors to core values and goals is a more effective motivator for many people than focusing solely on health outcomes.

For the growing number of adult patients with multiple chronic conditions (MCC), the benefits of effectively eliciting and incorporating their core values into care planning and decisions may be especially great. As Mary Tinetti has noted, ${ }^{4}$ the most common chronic condition experienced by adults is multimorbidity: the coexistence of multiple chronic diseases or conditions. Almost three in four adults aged 65 years and older have multiple chronic conditions, as do one in four adults younger than $65^{5}$ And these numbers are growing dramatically. Individuals with MCC frequently face numerous - and often conflicting - treatment choices that they need to weigh in light of their own unique needs, risks, priorities, and goals. It is thus crucial for their providers to be skilled at helping them identify and communicate what values and goals are most important to them for their health and wellbeing.

Yet, in spite of a growing recognition of the importance of incorporating patients' goals and values in care decisions, no best practices have yet been established for eliciting these from patients during care discussions. ${ }^{6}$ Moreover, there is limited empirical evidence regarding the key values of different patient populations and the most effective methods for eliciting and clarifying these values. Most values clarification tools developed for clinical practice to date focus on specific treatment decisions, and few of these methods have been evaluated experimentally to determine the effect of different approaches on values congruence and longer-term outcomes. ${ }^{7}$

Given this paucity of empirical data on patient values and approaches for effectively eliciting values, the qualitative study by Lim et al. in this issue of JGIM is especially welcome. The authors sought to identify what patients with MCC view as most important to their well-being and health. ${ }^{8}$ The researchers interviewed 31 older adults (mean age 68.7 years) with diabetes mellitus and at least two other common chronic conditions (depression, osteoarthritis, and coronary artery disease) who received care in a single integrated health care system in Washington state. The authors interviewed patients in their own homes, with optional inclusion of family 
members ( 18 spouses and 1 adult child participated). At least 1 week prior to each visit, the investigators sent the participant an instant camera with a written prompt to take up to 10 photos of "anything they considered most important to their wellbeing and health." The researchers then began each interview by asking participants to describe each photo and why they took it. Six core domains of personal values emerged from the interviews as most important for participants' well-being and health: 1) principles (beliefs and standards to live by, such as ideals, virtues, and aspirations); 2) relationships (social connections with important people and entities); 3) emotions (feeling moods, or states of being that are personal, embodied, and experiential); 4) activities (important pursuits for work, leisure, volunteering, improving health, and other purposes); 5) abilities (physical or mental capacities or skills); and 6) possessions (tangible objects and important spaces that have personal meaning). These personal values/goals were interrelated and rarely expressed as individual values in isolation.

This study extends earlier qualitative work among older multimorbid adults that has begun to explore health-related values as defined more broadly than simply preferences for individual diagnostic or therapeutic choices. For example, Naik et al. conducted semi-structured interviews with 146 multimorbid adults 12 months after they had been diagnosed with cancer and then again after treatment. ${ }^{9}$ The authors identified five distinct health-related values that guided these patients' conceptualization of health care goals and medical decisions: self-sufficiency, life enjoyment, connectedness and legacy, balancing quality and length of life, and engagement in care. As Lim et al. also argue, the identification of such taxonomies of key domains can help guide clinicians in formulating questions to explore patient values. As the values identified by Lim et al. were often interrelated - with one value subject to the influence of another, and all potentially affected by changes in health status - their work also demonstrates the importance of clearly identifying personal values so that trade-offs among values can be explored in discussions of care planning. Yet, as they also note, they only explored values among patients with diabetes and a limited number of other chronic conditions, who all received care in a high-quality integrated system. Their work is a useful guide for additional explorations with individuals from diverse socioeconomic and cultural backgrounds and who are grappling with other health conditions. This area of research is still nascent.

Lim et al. also raise the important question as to the optimal approaches for encouraging communication about patients' personal values during clinical encounters. As the authors note, many patients do not perceive personal values as pertinent to clinical conversations. We thus need innovative approaches to help capture personal values that patients may not think to disclose to their providers. The photo elicitation approach they used is intriguing - and its use in clinical care is worth further exploration. As almost all our patients now carry smartphone cameras almost everywhere (as do we clinicians), we certainly could encourage them to take photos to share with us images of what and who are most important to their health and well-being.
Several recent pilot studies of chronic disease self-management interventions have incorporated photovoice exercises to engage patients through the use of photographs and storytelling, with promising results. ${ }^{10}$ Their choice of the venue of people's homes for the discussion might also have facilitated patients' reflections on what is most important to them, further demonstrating both the potential research and clinical value of home visits.

Importantly, research such as this study by Lim et al. challenges us to identify and reflect on the range of values older adults with MCC have. An important next step is to develop and test approaches to elicit and incorporate patients' personal values into our ongoing discussions about care decisions and to negotiate how best to reconcile these values with available treatment options and desired outcomes. Finally, we need rigorous evaluations of whether and how adopting such approaches affects patients' health outcomes and attainment of goals. Does focusing more on "what matters" to patients indeed improve their health and well-being?

Corresponding Author: Michele Heisler, MD, MPA; Department of Health Behavior and Health Education, School of Public Health University of Michigan, Ann Arbor, MI, USA (e-mail: mheisler@umich. edu).

\section{Compliance with Ethical Standards:}

Conflict of Interest: The author declares that he/she does not have a conflict of interest.

\section{REFERENCES}

1. Committee on Quality of Health Care in America; Institute of Medicine. Crossing the quality chasm: a new health system for the 21 st century. Washington, DC: National Academy Press; 2001:6.

2. Ratanawongsa N, Karter AJ, Parker MM, et al. Communication and medication refill adherence: The Diabetes Study of Northern California. JAMA Intern Med. 2013;173(3):210-18.

3. Resnicow, K, McMaster F. Motivational Interviewing: moving from why to how with autonomy support. Intern $\mathrm{J}$ Behav Nutr Phys Activ. 2012;9:19.

4. Tinetti ME, Fried TR, Boyd CM. Designing health care for the most common chronic condition-multimorbidity. JAMA. 2012;307(23):24934. https://doi.org/10.1001/jama.2012.5265.

5. Anderson G. chronic care: making the case for ongoing care. Princeton, NJ: Robert Wood Johnson Foundation; 2010. Available at: http://www. rwjf.org/files/research/50968chronic.care.chartbook.pdf. Accessed August 22, 2017.

6. Witteman HO, Scherer LD, Gavaruzzi T, et al. Design features of explicit values clarification methods: a systematic review. Med Decis Making. 2016;0272989X15626397. https://doi.org/10.1177/ 0272989X15626397.

7. Witteman HO, Gavaruzzi T, Scherer LD, et al. Effects of design features of explicit values clarification methods: a systematic review. Med Decis Making. 2016;36:760-76.

8. Lim C, Berry ABL, Hirsch T, et al. Understanding what is most important to individuals with multiple chronic conditions: a qualitative study of patients' perspectives. J Gen Intern Med. https://doi.org/10. 1007/s11606-017-4154-3.

9. Naik AD, Martin LA, Moye J, Karel MJ. Health values and treatment goals of older, multimorbid adults facing life-threatening illness. J Am Geriatr Soc. 2016;64(3):625-31. https://doi.org/10.1111/jgs. 14027.

10. Baig AA, Benitz A, Locklin CA, et al. Picture Good Health: a churchbased self-management intervention among Latino adults with diabetes. J Gen Intern Med. 2015; 10:1481-90. 